\title{
Symmetries of the Continuous and Discrete Krichever-Novikov Equation ${ }^{\star}$
}

\author{
Decio LEVI ${ }^{\dagger}$, Pavel WINTERNITZ ${ }^{\ddagger}$ and Ravil I. YAMILOV ${ }^{\S}$ \\ † Dipartimento di Ingegneria Elettronica, Università degli Studi Roma Tre and Sezione INFN, \\ Roma Tre, Via della Vasca Navale 84, 00146 Roma, Italy \\ E-mail: levi@roma3.infn.it \\ URL: http://optow.ele.uniroma3.it/levi.html \\ $\ddagger$ Centre de recherches mathématiques and Département de mathématiques et de statistique, \\ Université de Montréal, C.P. 6128, succ. Centre-ville, H3C 3J7, Montréal (Québec), Canada \\ E-mail: wintern@crm.umontreal.ca \\ URL: http://www.crm.umontreal.ca/ wintern/ \\ $\S$ Ufa Institute of Mathematics, Russian Academy of Sciences, \\ 112 Chernyshevsky Street, Ufa 450008, Russian Federation \\ E-mail: RvlYamilov@matem.anrb.ru \\ URL: http://matem.anrb.ru/en/yamilovri
}

Received June 16, 2011, in final form October 15, 2011; Published online October 23, 2011 http://dx.doi.org/10.3842/SIGMA.2011.097

\begin{abstract}
A symmetry classification is performed for a class of differential-difference equations depending on 9 parameters. A 6-parameter subclass of these equations is an integrable discretization of the Krichever-Novikov equation. The dimension $n$ of the Lie point symmetry algebra satisfies $1 \leq n \leq 5$. The highest dimensions, namely $n=5$ and $n=4$ occur only in the integrable cases.
\end{abstract}

Key words: symmetry classification; integrable PDEs; integrable differential-difference equations

2010 Mathematics Subject Classification: 35B06; 35K25; 37K10; 39A14

\section{Introduction}

The Krichever-Novikov (KN) equation [7] is given by

$$
\dot{u}=\frac{1}{4} u_{x x x}-\frac{3}{8} \frac{\left(u_{x x}\right)^{2}}{u_{x}}+\frac{3}{2} \frac{P(u)}{u_{x}}, \quad \dot{u} \equiv u_{t},
$$

where $P(u)$ is an arbitrary fourth degree polynomial of its argument with constant coefficients. This is a nonlinear partial differential equation with 5 arbitrary constant parameters. Equation (1.1) first appeared in the study of the finite gap solutions of the Kadomtsev-Petviashvili equation $[8,7,21]$. For a special choice of $P(u)(1.1)$ reduces to the Korteweg-de Vries equation but for a generic polynomial no differential substitution exists reducing equation (1.1) to a KdV-type equation [24]. In [7, 5, 20], a zero-curvature representation was obtained for (1.1) involving $\operatorname{sl}(2)$ matrices. The Hamiltonian structure of (1.1) was analyzed and possible applications were reviewed in $[23,17]$. Bäcklund transformations have been constructed together with the nonlinear superposition formulae in [1]. The Lax representation was used in [23] to prove

${ }^{\star}$ This paper is a contribution to the Proceedings of the Conference "Symmetries and Integrability of Difference Equations (SIDE-9)" (June 14-18, 2010, Varna, Bulgaria). The full collection is available at http://www.emis.de/journals/SIGMA/SIDE-9.html 
that (1.1) has conservation laws. In [3] the authors considered a generalization of (1.1) in which the polynomial $P(u)$ is an arbitrary function of $u$ and studied its symmetry classification.

In 1983 Yamilov [30] introduced an integrable discretization of the Krichever-Novikov equation (the YdKN equation):

$$
\dot{u}_{n} \equiv u_{n, t}=f_{n}=\frac{S_{n}}{u_{n+1}-u_{n-1}},
$$

where the polynomial $S_{n}$ is given by

$$
\begin{aligned}
& S_{n}=P_{n} u_{n+1} u_{n-1}+Q_{n}\left(u_{n+1}+u_{n-1}\right)+R_{n}, \\
& P_{n}=\alpha u_{n}^{2}+2 \beta u_{n}+\gamma, \quad Q_{n}=\beta u_{n}^{2}+\lambda u_{n}+\delta, \quad R_{n}=\gamma u_{n}^{2}+2 \delta u_{n}+\omega .
\end{aligned}
$$

This is a differential-difference equation with 6 arbitrary constant parameters. By carrying out the continuous limit, we get the Krichever-Novikov equation (1.1) [30] (see Section 2 below).

The YdKN equation has been obtained as a result of a classification of differential-difference equations of the form $\dot{u}_{n}=f\left(u_{n-1}, u_{n}, u_{n+1}\right)$ with no explicit $n$ and $t$ dependence [30,31] that allow at least two conservation laws (or one conservation law and one generalized symmetry) of a high enough order. In the general case, when all parameters are different from zero, $(1.2),(1.3)$ is the only example in the complete list of Volterra type equations which cannot be transformed by Miura transformations into the Volterra or Toda lattice equation [31]. Recently it has been observed that most of the known integrable discrete equations on square lattices are closely related to the YdKN equation in the sense that they generate Bäcklund transformations of the YdKN equation $[10,27,16]$. An $L-A$ pair for the YdKN equation has been constructed in [27].

A generalization of the YdKN equation (GYdKN) introduced by D. Levi and R. Yamilov in [15] has the same form (1.2), (1.3), but with $n$-dependent coefficients:

$$
\begin{aligned}
& P_{n}=\alpha u_{n}^{2}+2 \beta_{n} u_{n}+\gamma_{n}, \quad Q_{n}=\beta_{n+1} u_{n}^{2}+\lambda u_{n}+\delta_{n+1}, \\
& R_{n}=\gamma_{n+1} u_{n}^{2}+2 \delta_{n} u_{n}+\omega .
\end{aligned}
$$

Here $\beta_{n}, \gamma_{n}, \delta_{n}$ are two-periodic, i.e. can be written in the form

$$
\beta_{n}=\beta+\hat{\beta}(-1)^{n}, \quad \gamma_{n}=\gamma+\hat{\gamma}(-1)^{n}, \quad \delta_{n}=\delta+\hat{\delta}(-1)^{n} .
$$

Thus the GYdKN equation depends on 9 arbitrary constant parameters. It has been shown in [15] that the GYdKN equation satisfies the lowest integrability conditions in the generalized symmetry classification of Volterra type equations. Both YdKN and GYdKN equations are integrable in the sense that they possess master symmetries [2] and therefore they have infinite hierarchies of generalized symmetries and conservation laws. The GYdKN equation is also closely related to non-autonomous discrete equations on square lattices [29]. It is worth mentioning here that this generalization does not allow a continuous limit to the Krichever-Novikov equation or any of its generalizations.

Extensions of the YdKN, which in the continuous limit reduce to the KN equation or its generalizations can be obtained by choosing $P_{n}, Q_{n}$ and $R_{n}$ as arbitrary $t$-independent functions of $u_{n}$. An interesting extension of the YdKN equation is given by the equation

$$
\begin{aligned}
& u_{n, t} \equiv \dot{u}_{n}=\frac{P\left(u_{n}\right) u_{n+1} u_{n-1}+Q\left(u_{n}\right)\left(u_{n+1}+u_{n-1}\right)+R\left(u_{n}\right)}{u_{n+1}-u_{n-1}} \\
& P\left(u_{n}\right)=\alpha u_{n}^{2}+2 \beta u_{n}+\gamma, \quad Q\left(u_{n}\right)=\hat{\beta} u_{n}^{2}+\lambda u_{n}+\delta, \quad R\left(u_{n}\right)=\hat{\gamma} u_{n}^{2}+2 \hat{\delta} u_{n}+\omega
\end{aligned}
$$

where $\alpha, \ldots, \omega$ are 9 real constants, at least one of them nonzero. We will call (1.6) the EYdKN (extended YdKN). Like the GYdKN the EYdKN equation depends on 9 constant coefficients. By choosing $\hat{\beta}=\beta, \hat{\gamma}=\gamma, \hat{\delta}=\delta$ it reduces to the YdKN equation. 
In the following we are going to carry out the point symmetry classification for all particular cases of the EYdKN equation. These are differential-difference equations and for them we will use the theory of symmetries of difference equations as presented in $[4,11,13,26,14]$. Due to its complication we present here just one example of an equation belonging to the GYdKN class which possesses a nontrivial point symmetry algebra.

In Section 2 we first take the continous limit of a generalized YdKN equation and then calculate the Lie point symmetries of the obtained (continuous) generalized Krichever-Novikov equation (1.1) in which $f(u) \equiv P(u)$ is an arbitrary function. Sections 3 and 4 are devoted to a symmetry classification of the EYdKN equation for which $P\left(u_{n}\right), Q\left(u_{n}\right)$ and $R\left(u_{n}\right)$ are restricted to being second order polynomials. This includes the integrable YdKN equation as a subcase. Some conclusions and future outlook are presented in Section 5.

\section{Continuous limit of a generalized YdKN equation and its Lie point symmetries}

\subsection{The continuous limit}

Let us look for the continuous limit of a generalization of the YdKN equation (1.2), (1.3). Here, for the sake of simplicity of notation we take $P\left(u_{n}\right), Q\left(u_{n}\right)$ and $R\left(u_{n}\right)$ as arbitrary functions of their argument. We carry out the continuous limit generalizing the procedure used in [30].

First of all we redefine the functions $P\left(u_{n}\right), Q\left(u_{n}\right)$ and $R\left(u_{n}\right)$

$$
P\left(u_{n}\right)=\tilde{P}\left(u_{n}\right)+k, \quad Q\left(u_{n}\right)=\tilde{Q}\left(u_{n}\right)-k u_{n}, \quad R\left(u_{n}\right)=\tilde{R}\left(u_{n}\right)+k u_{n}^{2},
$$

where $k$ is an arbitrary constant. We introduce a small parameter $h$, the lattice spacing, by putting

$$
\begin{aligned}
& \tilde{P}\left(u_{n}\right)=2 h F(v(x, t)), \quad \tilde{Q}\left(u_{n}\right)=2 h G(v(x, t)), \quad \tilde{R}\left(u_{n}\right)=2 h H(v(x, t)), \\
& u_{n}(t)=v(x, t), \quad x=n h+6 \frac{t}{h^{2}}, \quad k=-\frac{12}{h^{3}} .
\end{aligned}
$$

Taking the limit, $h \rightarrow 0$ and $n \rightarrow \infty$ with $n h$ finite, we get

$$
v_{t}=v_{x x x}-\frac{3}{2} \frac{v_{x x}^{2}}{v_{x}}+\frac{v^{2} F(v)+2 v G(v)+H(v)}{v_{x}}+\mathcal{O}\left(h^{2}\right) .
$$

Putting $v^{2} F(v)+2 v G(v)+H(v)=f(v)$ and replacing $v(x, t)$ by $u(x, t)$ we obtain the "generalized Krichever-Novikov equation"

$$
u_{t}=u_{x x x}-\frac{3}{2} \frac{\left(u_{x x}\right)^{2}}{u_{x}}+\frac{f(u)}{u_{x}} .
$$

Rescaling and restricting the arbitrary function $f(u)$ to a fourth order polynomial we obtain the Krichever-Novikov equation (1.1).

\subsection{Lie point symmetries of the continuous generalized Krichever-Novikov equation}

For comparison with the extended YdKN equation (1.7) we present a symmetry classification of (2.1), thus completing the partial classification performed in [3].

Equation (2.1) is form-invariant under "allowed transformations" that only change the form of $f(u)$. These include Möbius transformations of the dependent variable $u$ and a simultaneous rescaling of $x$ and $t$

$$
u=\frac{\alpha \tilde{u}+\beta}{\gamma \tilde{u}+\delta}, \quad \alpha \delta-\beta \delta \neq 0, \quad t=k^{3} \tilde{t}, \quad x=k \tilde{x} .
$$


The function $f(u)$ transforms into

$$
\tilde{f}(\tilde{u})=\frac{k^{4}}{(\alpha \delta-\beta \gamma)^{2}} f\left(\frac{\alpha u+\beta}{\gamma u+\delta}\right)(\gamma u+\delta)^{4}
$$

We shall classify (2.1) into symmetry classes under the action of the group of allowed transformations (2.2). The "group of allowed transformations" is sometimes also called the "equivalence group" of the equation.

We write a general element of the symmetry algebra of (2.1) in the form

$$
X=\tau(x, t, u) \partial_{t}+\xi(x, t, u) \partial_{x}+\phi(x, t, u) \partial_{u}
$$

Requiring that the third prolongation $\mathrm{pr}^{(3)} X$ of (2.4) should annihilate (2.1) on the solution set, we obtain 9 determining equations for the coefficients $\tau, \xi$ and $\phi$. The first 8 of them are elementary and imply

$$
\tau=\tau_{1} t+\tau_{0}, \quad \xi=\frac{1}{3} \tau_{1} x+\xi_{0}, \quad \phi=\phi_{2} u^{2}+\phi_{1} u+\phi_{0},
$$

where $\tau_{0}, \tau_{1}, \xi_{0}, \phi_{2}, \phi_{1}$ and $\phi_{0}$ are constants. The remaining determining equation implies that the function $f(u)$ figuring in (2.1) must satisfy the following first order ODE:

$$
\left(\phi_{2} u^{2}+\phi_{1} u+\phi_{0}\right) \frac{d f}{d u}+\left[-4 \phi_{2} u+\frac{4}{3} \tau_{1}-2 \phi_{1}\right] f=0 .
$$

A symmetry analysis of (2.1) thus boils down to analyzing all possible solutions of (2.5).

First of all (2.5) does not contain $\tau_{0}$ and $\xi_{0}$. This is just a reflection of the obvious fact that (2.1) does not depend explicitly on $t$ and $x$ and is hence invariant under time and space translations for any function $f(u)$. They are generated by

$$
P_{0}=\partial_{t}, \quad P_{1}=\partial_{x}
$$

respectively.

Let us now assume that at least one of the coefficients $\phi_{0}, \phi_{1}, \phi_{2}$ or $\tau_{1}$ is nonzero. In Table 1 we present representatives of all classes of functions $f(u)$ for which the symmetry algebra $L$ of (2.1) is larger than (2.6). We have $2<\operatorname{dim} L \leq 6$ in all cases. The classification is under the allowed transformations (2.2), (2.3). The following cases occur, depending on the properties of the polynomial

$$
\phi(u)=\phi_{2} u^{2}+\phi_{1} u+\phi_{0} .
$$

1. $\phi_{2} \neq 0, \phi(u)=0$ has complex roots $u_{1,2}=r \pm i s, s>0$. After an allowed transformation the solution is

$$
f(u)=f_{0}\left(1+u^{2}\right)^{2} e^{p \arctan u}, \quad f_{0}= \pm 1,
$$

with $p \in \mathbb{R} ; p=0$ is a special case.

2. $\phi_{2} \neq 0, \phi(u)=0$ has two real roots $u_{1}<u_{2}$,

$$
f(u)=f_{0}(u+1)^{p}(u-1)^{4-p}, \quad f_{0}= \pm 1 .
$$

Since $p$ and $4-p$ are equivalent, we can restrict to the case $2 \leq p<\infty$. The case $p=2$ is again special. For $p=2,3,4(2.7)$ is a fourth order polynomial. 
Table 1. Symmetry classification of the continuous generalized Krichever-Novikov equation; $f_{0}= \pm 1$, see Section 2 for notation.

\begin{tabular}{|c|c|c|c|}
\hline$N_{0}$ & $f(u)$ & $\operatorname{dim} L$ & Basis elements of symmetry algebra $L$ \\
\hline \hline 1 & 0 & 6 & $P_{0}, P_{1}, D, U_{0}, U_{1}, U_{2}$ \\
\hline 2 & $f_{0}$ & 4 & $P_{0}, P_{1}, D+\frac{2}{3} U_{1}, U_{0}$ \\
\hline 3 & $f_{0} u^{p}$ & 3 & $P_{0}, P_{1},(p-2) D-\frac{4}{3} U_{1}, 2 \leq p<\infty$ \\
\hline 4 & $f_{0} e^{u}$ & 3 & $P_{0}, P_{1}, D-\frac{4}{3} U_{0}$ \\
\hline 5 & $f_{0}\left(u^{2}+1\right)^{2} e^{p \arctan u}$ & 3 & $P_{0}, P_{1}, p D-\frac{4}{3}\left(U_{2}+U_{0}\right)$ \\
\hline 6 & $f_{0}(u+1)^{p}(u-1)^{4-p}$ & 3 & $P_{0}, P_{1},(p-2) D-\frac{2}{3}\left(U_{2}-U_{0}\right) 2 \leq p<\infty$ \\
\hline
\end{tabular}

3. $\phi_{2} \neq 0, \phi(u)=0$ has a double root and we shift it to $u_{1}=u_{2}=0$. We obtain $f(u)=$ $f_{0} u^{4} e^{-\frac{p}{u}}$. An allowed transformation takes this into

$$
f(u)=f_{0} e^{u}, \quad f_{0}= \pm 1
$$

4. $\phi_{2}=0, \phi_{1} \neq 0$ We obtain

$$
f(u)=f_{0} u^{p}, \quad f_{0}= \pm 1 .
$$

Under an allowed transformation we have $p \rightarrow 4-p$ so we can restrict $p$ to $2 \leq p<\infty$. The case $p=4$ is equivalent to $f(u)=f_{0}$.

5. $\phi_{2}=0, \phi_{1}=0, \phi_{0} \neq 0$. We reobtain $(2.8)$ or $f(u)=f_{0}$.

In Table 1 we give the functions $f(u)$ in column 2 and the basis elements of the Lie algebra in column 4. Throughout we have $f_{0}= \pm 1$ and we use the notation (2.6) and

$$
D=t \partial_{t}+\frac{1}{3} x \partial_{x}, \quad U_{0}=\partial_{u}, \quad U_{1}=u \partial_{u}, \quad U_{2}=u^{2} \partial_{u}
$$

The Lie algebras in cases $3,4,5$ and 6 of Table 1 are all solvable with $\left\{P_{0}, P_{1}\right\}$ as their nilradical. The values $p=2$ in case 3 and 6 and $p=0$ in case 5 are special as the Lie algebra for these values contracts to an Abelian one.

\section{Symmetry structure of the extended YdKN equation}

\subsection{Allowed transformations}

First of all we notice that (1.6), (1.7) is form-invariant under the Möbius transformation

$$
u_{n} \rightarrow \tilde{u}_{n}=\frac{\eta_{1} u_{n}+\eta_{2}}{\eta_{3} u_{n}+\eta_{4}}, \quad \Delta=\eta_{1} \eta_{4}-\eta_{2} \eta_{3}= \pm 1
$$

where $\eta_{i}, i=1, \ldots, 4$ are arbitrary real constants. All such $S L(2, \mathbb{R})$ transformations can be induced by combinations of translations $\tilde{u}_{n}=u_{n}+\kappa$, dilations $\tilde{u}_{n}=\kappa u_{n}$ and the inversion $\tilde{u}_{n}=1 / u_{n}$. Explicitly the coefficients $\alpha, \ldots, \omega$ of (1.7) transform under a translation into

$$
\begin{aligned}
& \alpha^{*}=\alpha, \quad \beta^{*}=\beta+\alpha \kappa, \quad \hat{\beta}^{*}=\hat{\beta}+\alpha \kappa, \quad \gamma^{*}=\gamma+2 \beta \kappa+\alpha \kappa^{2}, \\
& \hat{\gamma}^{*}=\hat{\gamma}+2 \hat{\beta} \kappa+\alpha \kappa^{2}, \quad \lambda^{*}=\lambda+2(\beta+\hat{\beta}) \kappa+2 \alpha \kappa^{2}, \\
& \hat{\delta}^{*}=\hat{\delta}+(\hat{\gamma}+\lambda) \kappa+(\beta+2 \hat{\beta}) \kappa^{2}+\alpha \kappa^{3}, \quad \delta^{*}=\delta+(\gamma+\lambda) \kappa+(2 \beta+\hat{\beta}) \kappa^{2}+\alpha \kappa^{3},
\end{aligned}
$$




$$
\omega^{*}=\omega+2(\delta+\hat{\delta}) \kappa+(\gamma+\hat{\gamma}+2 \lambda) \kappa^{2}+2(\beta+\hat{\beta}) \kappa^{3}+\alpha \kappa^{4},
$$

under a dilation into

$$
\begin{array}{lll}
\alpha^{*}=\alpha \kappa^{2}, & \beta^{*}=\beta \kappa, & \hat{\beta}^{*}=\hat{\beta} \kappa, \quad \gamma^{*}=\gamma, \quad \hat{\gamma}^{*}=\hat{\gamma}, \quad \lambda^{*}=\lambda, \\
\delta^{*}=\delta / \kappa, & \hat{\delta}^{*}=\hat{\delta} / \kappa, \quad \omega^{*}=\omega / \kappa^{2}, &
\end{array}
$$

and under the inversion into

$$
\begin{aligned}
& \alpha^{*}=\omega, \quad \beta^{*}=\hat{\delta}, \quad \hat{\beta}^{*}=\delta, \quad \gamma^{*}=\hat{\gamma}, \quad \hat{\gamma}^{*}=\gamma, \quad \lambda^{*}=\lambda, \\
& \delta^{*}=\hat{\beta}, \quad \hat{\delta}^{*}=\beta, \quad \omega^{*}=\alpha .
\end{aligned}
$$

Equation (1.6) is also form-invariant under dilation of time (and invariant under time translation).

\subsection{Theorems simplifying the symmetry classification}

First of all, we shall show that we can restrict the study of the EYdKN equation to the case $P\left(u_{n}\right) \neq 0$ in (1.7) and that this can be split into precisely 3 subcases.

Theorem 1. Using the Möbius transformation (3.1) we can reduce the EYdKN equation (1.6), (1.7) for $\left(P_{n}, Q_{n}, R_{n}\right) \neq(0,0,0)$ to one of the 3 following cases:

1. $\alpha=1, \beta=0$;

2. $\alpha=0, \beta=1, \gamma=0$;

$$
\beta+\hat{\beta}=\delta+\hat{\delta}=\gamma+\hat{\gamma}+2 \lambda=\omega=0
$$

3. $\alpha=0, \beta=0, \gamma=1$

$$
\hat{\beta}=\delta=\hat{\delta}=\gamma+\lambda=\hat{\gamma}+\lambda=\omega=0 .
$$

In all cases we have $P\left(u_{n}\right) \neq 0$.

Proof. If $\alpha \neq 0$ we can scale it to $\alpha=1$ and then transform $\beta$ into $\beta=0$ by a translation of $u_{n}$.

Now assume $\alpha=0, \beta \neq 0$. Up to Möbius transformations we must also assume $\alpha^{*}=0$ in (3.2). This imposes the conditions (3.3) on the other coefficients in the EYdKN equation, otherwise we can always chose $\kappa$ to obtain $\alpha^{*} \neq 0$. For $\beta \neq 0$ we can again dilate to obtain $\beta=1$ and translate $u_{n}$ to obtain $\gamma=0$.

The third case corresponds to $\alpha=\beta=0, \gamma \neq 0$ and we dilate to obtain $\gamma=1$. Conditions (3.4) follow from the requirement $\alpha^{*}=0, \beta^{*}=0$ for all values of $\eta_{i}, i=1, \ldots, 4$ in the Möbius transformation.

Finally, if we impose $\alpha=\beta=\gamma=0$ and also $\alpha^{*}=\beta^{*}=\gamma^{*}=0$ for all values $\eta_{i}, i=1, \ldots, 4$ we obtain not only $P\left(u_{n}\right)=0$ but also $Q\left(u_{n}\right)=R\left(u_{n}\right)=0$, i.e. (1.6) is trivial.

Comment. A further scaling of one more parameter can be achieved using a dilation of time $t$. This can provide simplifications which will be discussed below in Section 4 in each specific case.

Theorem 2. The Lie algebra of local Lie point symmetries of the EYdKN equation with $\left(P_{n}, Q_{n}, R_{n}\right) \neq(0,0,0)$ consists of vector fields of the general form

$$
X=\tau(t) \partial_{t}+\phi_{n}\left(t, u_{n}\right) \partial_{u_{n}}
$$


with

$$
\begin{aligned}
& \tau=\tau_{0}+\tau_{1} t, \quad \phi_{n}=a_{n}+b_{n} u_{n}+c_{n} u_{n}^{2}, \\
& a_{n}=a+\hat{a}(-1)^{n}, \quad b_{n}=b+\hat{b}(-1)^{n}, \quad c_{n}=c+\hat{c}(-1)^{n},
\end{aligned}
$$

where $\tau_{0}, \tau_{1}, a, \hat{a}, b, \hat{b}, c$ and $\hat{c}$ are constants.

Proof. In a previous article [14] we have shown that for a large class of differential-difference equations, including the EYdKN equation, the vector field corresponding to Lie point symmetries must have the form (3.5), in particular $\tau(t)$ does not depend on $n$ or $u_{n}$. The first prolongation of $X$ to be applied to (1.6) is

$$
\operatorname{pr} X=\tau(t) \partial_{t}+\sum_{j=n-1}^{n+1} \phi_{j}\left(t, u_{j}\right) \partial_{u_{j}}+\phi_{n}^{(1)} \partial_{\dot{u}_{n}}, \quad \phi_{n}^{(1)}=D_{t} \phi_{n}\left(t, u_{n}\right)-\left[D_{t} \tau(t)\right] \dot{u}_{n}
$$

where $D_{t}$ is the total derivative operator. Applying $\operatorname{pr} X$ to the equation and requiring the result to be zero on the solution set, we obtain the determining equation

$$
\begin{aligned}
& \phi_{n, t}\left(u_{n+1}-u_{n-1}\right)^{2}+\left(\phi_{n, u_{n}}-\dot{\tau}\right)\left[P u_{n+1} u_{n-1}+Q\left(u_{n+1}+u_{n-1}\right)+R\right]\left(u_{n+1}-u_{n-1}\right) \\
& \quad-\phi_{n}\left[P_{, u_{n}} u_{n+1} u_{n-1}+Q_{, u_{n}}\left(u_{n+1}+u_{n-1}\right)+R_{, u_{n}}\right]\left(u_{n+1}-u_{n-1}\right) \\
& \quad=\phi_{n-1}\left[P u_{n+1}^{2}+2 Q u_{n+1}+R\right]-\phi_{n+1}\left[P u_{n-1}^{2}+2 Q u_{n-1}+R\right] .
\end{aligned}
$$

From Theorem 1 we know that we only need to consider the case $P\left(u_{n}\right) \neq 0$. Applying the fourth derivative $\partial_{u_{n+1}}^{2} \partial_{u_{n-1}}^{2}$ to (3.8) and dividing by $P_{n}$ we obtain

$$
\phi_{n+1, u_{n+1} u_{n+1}}-\phi_{n-1, u_{n-1} u_{n-1}}=0 .
$$

This implies

$$
\phi_{n}=a_{n}+b_{n} u_{n}+c_{n} u_{n}^{2}, \quad c_{n}=c+\hat{c}(-1)^{n} .
$$

Putting (3.9) back into (3.8) and comparing independent terms we obtain

$$
\ddot{\tau}=0, \quad a_{n+1}=a_{n-1}, \quad b_{n+1}=b_{n-1},
$$

where all coefficients are time independent. This completes the proof of Theorem 2.

\subsection{The determining equations}

Let us now return to (3.8) and substitute into it the expression (3.6) and (3.7) for $\tau$ and $\phi_{n}$, as well as (1.7) for $P, Q$ and $R$. The expressions multiplying $\left(u_{n+1}\right)^{k}\left(u_{n-1}\right)^{\ell}$ for different values of $k$ and $\ell$ must vanish separately. This will provide us with two sets of linear algebraic homogeneous equations, one for the vector $\vec{v}_{1}=\left(a, b, c, \tau_{1}\right)$, the other for $\vec{v}_{2}=(\hat{a}, \hat{b}, \hat{c})$. The coefficient $\tau_{0}$ does not figure anywhere, so

$$
P_{0}=\frac{\partial}{\partial t}
$$

is always an element of the algebra.

We write these two matrix determining equations as

$$
\hat{M}_{1} \vec{v}_{1}=\overrightarrow{0}, \quad \hat{M}_{2} \vec{v}_{2}=\overrightarrow{0}
$$


and denote

$$
r_{1}=\operatorname{rank} \hat{M}_{1}, \quad r_{2}=\operatorname{rank} \hat{M}_{2} .
$$

The dimension of the symmetry algebra of the EYdKN equation will be

$$
\operatorname{dim} L=8-\left(r_{1}+r_{2}\right),
$$

and in view of Theorem 1 we need only to consider the case $P \neq 0$. The two matrices involved are

$$
\begin{aligned}
\hat{M}_{1}= & \left(\begin{array}{cccc}
\alpha & \beta & -(\lambda+\gamma) & \beta \\
\alpha & \hat{\beta} & -(\lambda+\hat{\gamma}) & \hat{\beta} \\
0 & 2 \alpha & -2(\beta+\hat{\beta}) & \alpha \\
2 \beta & 0 & -2 \delta & \gamma \\
-2(\beta+\hat{\beta}) & 0 & 2(\delta+\hat{\delta}) & -\lambda \\
2 \hat{\beta} & 0 & -2 \hat{\delta} & \hat{\gamma} \\
-(\lambda+\gamma) & \delta & \omega & -\delta \\
-(\lambda+\hat{\gamma}) & \hat{\delta} & \omega & -\hat{\delta} \\
2(\delta+\hat{\delta}) & -2 \omega & 0 & \omega
\end{array}\right), \\
\hat{M}_{2}= & \left(\begin{array}{ccc}
\alpha & -\beta & -(\gamma-\lambda) \\
\alpha & -\hat{\beta} & -(\hat{\gamma}-\lambda) \\
\beta & -\gamma & \delta \\
\hat{\beta} & -\hat{\gamma} & \hat{\delta} \\
\beta-\hat{\beta} & 0 & \delta-\hat{\delta} \\
\gamma-\lambda & \delta & -\omega \\
\hat{\gamma}-\lambda & \hat{\delta} & -\omega \\
\delta-\hat{\delta} & 0 & 0 \\
0 & 0 & \beta-\hat{\beta}
\end{array}\right) .
\end{aligned}
$$

\section{Symmetry classification for the EYdKN equation}

\subsection{General comments}

Let us introduce a notation for the matrix of coefficients of the EYdKN equation (1.6), (1.7):

$$
K=\left(\begin{array}{ccc}
\alpha & \beta & \gamma \\
\hat{\beta} & \lambda & \delta \\
\hat{\gamma} & \hat{\delta} & \omega
\end{array}\right)
$$

We will analyze the possible ranks of the two matrices $\hat{M}_{1}$ and $\hat{M}_{2}$ of (3.10) and (3.11), as functions of the coefficients in $K$. We shall first determine all cases when the ranks $r_{1}$ and $r_{2}$ satisfy $r_{1}+r_{2} \leq 5$ so that the symmetry algebra $L$ has dimension $3 \leq \operatorname{dim} L \leq 5$. Separately we list all cases when we have $\operatorname{dim} L=2$.

\subsection{Symmetry algebras with $\operatorname{dim} L \geq 3$ and $\alpha \neq 0$}

We take $\alpha=1, \beta=0$. Inspecting the matrices $\hat{M}_{1}$ and $\hat{M}_{2}$ of (3.10) and (3.11) we see that their ranks satisfy

$$
2 \leq r_{1} \leq 4, \quad 1 \leq r_{2} \leq 3
$$


The cases relevant for this section are:

\section{$\operatorname{dim} L=5$.}

$r_{1}=2, r_{2}=1, \alpha=1, \beta=\hat{\beta}=\gamma=\hat{\gamma}=\delta=\hat{\delta}=\lambda=\omega=0$.

$$
\begin{aligned}
& K=\left(\begin{array}{ccc}
1 & 0 & 0 \\
0 & 0 & 0 \\
0 & 0 & 0
\end{array}\right), \quad \dot{u}_{n}=\frac{u_{n}^{2} u_{n+1} u_{n-1}}{u_{n+1}-u_{n-1}}, \\
& X_{0}=\partial_{t}, \quad X_{1}=2 t \partial_{t}-u_{n} \partial_{u_{n}}, \quad X_{2}=(-1)^{n} u_{n} \partial_{u_{n}}, \\
& X_{3}=u_{n}^{2} \partial_{u_{n}}, \quad X_{4}=(-1)^{n} u_{n}^{2} \partial_{u_{n}} .
\end{aligned}
$$

This is a 5 -dimensional solvable Lie algebra with Abelian nilradical $\left\{X_{0}, X_{3}, X_{4}\right\}$. The nonnilpotent elements $\left\{X_{1}, X_{2}\right\}$ commute and have a diagonal action on the nilradical. We mention that the allowed transformation $u_{n} \rightarrow \frac{1}{u_{n}}$ takes (4.1) into the differential-difference equation

$$
\dot{u}_{n}=\frac{1}{u_{n+1}-u_{n-1}}
$$

sometimes called the discrete $\mathrm{KdV}$ equation [18, 9, 10, 28, 22].

$$
\begin{aligned}
& \operatorname{dim} \boldsymbol{L}=\mathbf{4} \\
& r_{1}=3, r_{2}=1, \alpha=1, \beta=\hat{\beta}=\gamma=\hat{\gamma}=\delta=\hat{\delta}=0, \omega=\lambda^{2}, \lambda= \pm 1 . \\
& K=\left(\begin{array}{ccc}
1 & 0 & 0 \\
0 & \lambda & 0 \\
0 & 0 & 1
\end{array}\right), \quad \dot{u}_{n}=\frac{u_{n}^{2} u_{n+1} u_{n-1}+\lambda u_{n}\left(u_{n+1}+u_{n-1}\right)+\lambda^{2}}{u_{n+1}-u_{n-1}}, \\
& X_{0}=\partial_{t}, \quad X_{1}=(-1)^{n} u_{n} \partial_{u_{n}}, \quad X_{2}=(-1)^{n}\left(u_{n}^{2}-\lambda\right) \partial_{u_{n}}, \quad X_{3}=\left(u_{n}^{2}+\lambda\right) \partial_{u_{n}} .
\end{aligned}
$$

The algebra is reductive and isomorphic to $g l(2, \mathbb{R})$.

$\operatorname{dim} \boldsymbol{L}=\mathbf{3}$. We obtain two cases, namely:

1. $r_{1}=3, r_{2}=2, \alpha=1, \beta=\hat{\beta}=\delta=\hat{\delta}=\lambda=\omega=0, \gamma=\hat{\gamma}= \pm 1$.

$$
\begin{aligned}
& K=\left(\begin{array}{ccc}
1 & 0 & \gamma \\
0 & 0 & 0 \\
\gamma & 0 & 0
\end{array}\right), \quad \dot{u}_{n}=\frac{\left(u_{n}^{2}+\gamma\right)\left(u_{n+1} u_{n-1}+\gamma\right)}{u_{n+1}-u_{n-1}}, \\
& X_{0}=\partial_{t}, \quad X_{1}=(-1)^{n}\left(u_{n}^{2}+\gamma\right) \partial_{u_{n}}, \quad X_{2}=\left(u_{n}^{2}+\gamma\right) \partial_{u_{n}} .
\end{aligned}
$$

The algebra is Abelian.

2. $r_{1}=3, r_{2}=2, \alpha=1, \beta=\hat{\beta}=0, \gamma=\hat{\gamma}=-\mu^{2}, \delta=\hat{\delta}=2 \mu^{3}, \lambda=-2 \mu^{2}$ and $\omega=-3 \mu^{4}$.

$$
\begin{aligned}
& K=\left(\begin{array}{ccc}
1 & 0 & -\mu^{2} \\
0 & -2 \mu^{2} & 2 \mu^{3} \\
-\mu^{2} & 2 \mu^{3} & -3 \mu^{4}
\end{array}\right), \\
& \dot{u}_{n}=\frac{\left(u_{n}-\mu\right)\left[\left(u_{n}+\mu\right) u_{n+1} u_{n-1}-2 \mu^{2}\left(u_{n+1}+u_{n-1}\right)-\mu^{2}\left(u_{n}-3 \mu\right)\right]}{u_{n+1}-u_{n-1}}, \\
& X_{0}=\partial_{t}, \quad X_{1}=(-1)^{n}\left(u_{n}-\mu\right)^{2} \partial_{u_{n}}, \quad X_{2}=t \partial_{t}-\frac{1}{4 \mu}\left(u_{n}+3 \mu\right)\left(u_{n}-\mu\right) \partial_{u_{n}} .
\end{aligned}
$$

The algebra is solvable with an Abelian nilradical $\left\{X_{0}, X_{1}\right\}$. 


\subsection{Symmetry algebras with $\operatorname{dim} L \geq 3$ and $\alpha=0, \beta \neq 0$}

In this case we take $\beta=1, \gamma=0$ and in view of (3.3) we have

$$
\alpha=0, \quad \beta=1, \quad \gamma=0, \quad \hat{\beta}=-1, \quad \hat{\delta}=-\delta, \quad \hat{\gamma}=-2 \lambda, \quad \omega=0,
$$

so the matrix $K$ is

$$
K=\left(\begin{array}{ccc}
0 & 1 & 0 \\
-1 & \lambda & \delta \\
-2 \lambda & -\delta & 0
\end{array}\right)
$$

In this case the rank of $\hat{M}_{2}$ is always $r_{2}=3$ so we have $\hat{a}=\hat{b}=\hat{c}=0$ in (3.7). The dimension of the symmetry algebra is $\operatorname{dim} L=5-r_{1}$. The only case of interest here is $r_{1}=2$ and that requires $\lambda=\delta=0$. In this case we have

$\operatorname{dim} L=3$.

$r_{1}=2, r_{2}=3$. $\beta=1, \hat{\beta}=-1, \alpha=\gamma=\hat{\gamma}=\delta=\hat{\delta}=\lambda=\omega=0$.

$$
\begin{aligned}
& K=\left(\begin{array}{ccc}
0 & 1 & 0 \\
-1 & 0 & 0 \\
0 & 0 & 0
\end{array}\right), \quad \dot{u}_{n}=\frac{2 u_{n} u_{n+1} u_{n-1}-u_{n}^{2}\left(u_{n+1}+u_{n-1}\right)}{u_{n+1}-u_{n-1}}, \\
& X_{0}=\partial_{t}, \quad X_{1}=t \partial_{t}-u_{n} \partial_{u_{n}}, \quad X_{2}=u_{n}^{2} \partial_{u_{n}} .
\end{aligned}
$$

The algebra is solvable with an Abelian nilradical $\left\{X_{0}, X_{2}\right\}$.

\subsection{Symmetry algebras with $\operatorname{dim} L \geq 3$ and $\alpha=\beta=0, \gamma \neq 0$}

We normalize $\gamma$ to $\gamma=1$ by rescaling $t$ and have

$$
K=\left(\begin{array}{ccc}
0 & 0 & 1 \\
0 & -1 & 0 \\
1 & 0 & 0
\end{array}\right) \text {. }
$$

This leads to one further four-dimensional Lie algebra, namely

$$
\operatorname{dim} L=4 \text {. }
$$$$
r_{1}=1, r_{2}=3 \text {. }
$$

$$
\begin{aligned}
& \dot{u}_{n}=\frac{u_{n+1} u_{n-1}-u_{n}\left(u_{n+1}+u_{n-1}\right)+u_{n}^{2}}{u_{n+1}-u_{n-1}}, \\
& X_{0}=\partial_{t}, \quad X_{1}=\partial_{u_{n}}, \quad X_{2}=u_{n} \partial_{u_{n}}, \quad X_{3}=u_{n}^{2} \partial_{u_{n}} .
\end{aligned}
$$

The algebra is isomorphic to $g l(2, \mathbb{R})$.

\subsection{Symmetry algebras of dimension $\operatorname{dim} L=2$}

A symmetry algebra of dimension $\operatorname{dim} L=2$ will have one element $X$, in addition to $X_{0}=\partial_{t}$.

The element $X$ can have one of two forms: $X=\left(a+b u_{n}+c u_{n}^{2}\right) \partial_{u_{n}}+\tau_{1} \partial_{t}$ and it occurs for $r_{1}=3, r_{2}=3$, or $X=(-1)^{n}\left(\hat{a}+\hat{b} u_{n}+\hat{c} u_{n}^{2}\right) \partial_{u_{n}}$ for $r_{1}=4, r_{2}=2$. We shall consider the two cases separately, following the same branches as for $\operatorname{dim} L \geq 3$. 
I. $r_{1}=3, r_{2}=3$

Branch 1. $\alpha=1, \beta=0$

$\mathbf{I}_{1}, \gamma \neq \mathbf{0}$ (we can normalize it to $\gamma= \pm 1$ ). The matrix $\hat{M}_{1}$ is equivalent to

$$
\hat{M}_{1} \sim\left(\begin{array}{cccc}
1 & 0 & -(\lambda+\gamma) & 0 \\
0 & 1 & -\hat{\beta} & \frac{1}{2} \\
0 & 0 & -\frac{2 \delta}{\gamma} & 1
\end{array}\right)
$$

with all further rows vanishing in order for the rank to be $r_{1}=3$. This implies the following conditions on the parameters in the equation

$$
\begin{aligned}
& \gamma-\hat{\gamma}+\hat{\beta}^{2}+\frac{\delta \hat{\beta}}{\gamma}=0, \quad \delta+\hat{\delta}-\hat{\beta}(\lambda+\gamma)-\frac{\lambda \delta}{\gamma}=0, \\
& \omega-(\lambda+\gamma)^{2}+\hat{\beta} \delta-3 \frac{\delta^{2}}{\gamma}=0, \quad \omega-(\lambda+\gamma)(\lambda+\hat{\gamma})+\hat{\beta} \delta-3 \frac{\delta \hat{\delta}}{\gamma}=0, \\
& (\delta+\hat{\delta})(\lambda+\gamma)-\hat{\beta} \omega+\frac{2 \delta \omega}{\gamma}=0, \quad \delta(\gamma+\hat{\gamma}-\lambda)=0 .
\end{aligned}
$$

In order to obtain all symmetry algebras with $\operatorname{dim} L=2$ we must find all solutions of the system (4.7). From the last equation we obtain either $\delta=0$, or $\lambda=\gamma+\hat{\gamma}$. Thus the problem immediately branches in two. We then obtain $\hat{\gamma}, \hat{\delta}$ and $\omega$ from the first 3 equations. The remaining two equations provide nonlinear constraints on the remaining parameters. We shall not present the rather boring (computer assisted) analysis here, and only list the results. In each case we must make sure that we also have $r_{2}=3$ for the rank of $\hat{M}_{2}$.

$$
\begin{aligned}
& \text { 1. } K=\left(\begin{array}{ccc}
1 & 0 & \gamma \\
\hat{\beta} & \lambda & 0 \\
\gamma+\hat{\beta}^{2} & \hat{\beta}(\lambda+\gamma) & (\lambda+\gamma)^{2}
\end{array}\right), \quad(\hat{\beta}, \gamma, \lambda) \neq(0,0,0) \text {, } \\
& \dot{u}_{n}=\left\{\left(u_{n}^{2}+\gamma\right) u_{n+1} u_{n-1}+\left(\hat{\beta} u_{n}^{2}+\lambda u_{n}\right)\left(u_{n+1}+u_{n-1}\right)+\gamma u_{n}^{2}\right. \\
& \left.+\left(\hat{\beta} u_{n}+\lambda+\gamma\right)^{2}\right\} /\left(u_{n+1}-u_{n-1}\right), \\
& X=\left[\lambda+\gamma+\hat{\beta} u_{n}+u_{n}^{2}\right] \partial_{u_{n}} \text {. } \\
& \text { 2. } K=\left(\begin{array}{ccc}
1 & 0 & \gamma \\
0 & 2 \gamma & \delta \\
\gamma & \delta & -3 \gamma^{2}
\end{array}\right), \quad(\gamma, \delta) \neq(0,0), \quad \delta^{2}+4 \gamma^{3}=0, \quad \gamma<0 \text {, } \\
& \dot{u}_{n}=\frac{\left(u_{n}^{2}+\gamma\right) u_{n+1} u_{n-1}+\left(2 \gamma u_{n}+\delta\right)\left(u_{n+1}+u_{n-1}\right)+\gamma u_{n}^{2}+2 \delta u_{n}-3 \gamma^{2}}{u_{n+1}-u_{n-1}} \text {, } \\
& X=\frac{\gamma}{2 \delta}\left[\gamma-\frac{1}{2} u_{n}+u_{n}^{2}\right] \partial_{u_{n}}+t \partial_{t} . \\
& \text { 3. } K=\left(\begin{array}{ccc}
1 & 0 & \gamma \\
\hat{\beta} & \lambda & \delta \\
\hat{\gamma} & \hat{\delta} & \omega
\end{array}\right), \quad(\hat{\beta}, \delta) \neq(0,0), \quad \gamma<0, \\
& \hat{\beta}=2 \epsilon \sqrt{-\gamma}-\frac{\delta}{\gamma}, \quad \epsilon= \pm 1, \quad \hat{\gamma}=-3 \gamma+2 \epsilon \frac{\delta}{\sqrt{-\gamma}}, \\
& \hat{\delta}=2\left(\delta+\epsilon(-\gamma)^{3 / 2}\right), \quad \omega=\gamma^{2}-6 \epsilon \sqrt{-\gamma} \delta, \\
& \dot{u}_{n}=\frac{\left(u_{n}^{2}+\gamma\right) u_{n+1} u_{n-1}+\left(\hat{\beta} u_{n}^{2}+\lambda u_{n}+\delta\right)\left(u_{n+1}+u_{n-1}\right)+\hat{\gamma} u_{n}^{2}+\hat{\delta} u_{n}+\omega}{u_{n+1}-u_{n-1}}, \\
& X=t \partial_{t}+\left[(\lambda+\gamma) \frac{\gamma}{2 \delta}+\frac{1}{2}\left(\hat{\beta} \frac{\gamma}{\delta}-1\right) u_{n}+\frac{\gamma}{2 \delta} u_{n}^{2}\right] \partial_{u_{n}} .
\end{aligned}
$$


4. $K=\left(\begin{array}{ccc}1 & 0 & 0 \\ \hat{\beta} & \lambda & 0 \\ \hat{\beta}^{2} & \hat{\beta} \lambda & \lambda^{2}\end{array}\right), \quad \hat{\beta} \neq 0, \quad \lambda \neq \hat{\beta}^{2}$,

$$
\begin{aligned}
& \dot{u}_{n}=\frac{u_{n}^{2} u_{n+1} u_{n-1}+u_{n}\left(\hat{\beta} u_{n}+\lambda\right)\left(u_{n+1}+u_{n-1}\right)+\hat{\beta}^{2} u_{n}^{2}+2 \hat{\beta} \lambda u_{n}+\lambda^{2}}{u_{n+1}-u_{n-1}}, \\
& X=\left[\hat{\beta} \lambda+\hat{\beta} u_{n}+u_{n}^{2}\right] \partial_{u_{n}} .
\end{aligned}
$$

5. $K=\left(\begin{array}{ccc}1 & 0 & 0 \\ \hat{\beta} & 0 & 0 \\ 0 & 0 & 0\end{array}\right), \quad \hat{\beta} \neq 0$

$$
\dot{u}_{n}=\frac{u_{n}^{2} u_{n+1} u_{n-1}+\hat{\beta} u_{n}^{2}\left(u_{n+1}+u_{n-1}\right)}{u_{n+1}-u_{n-1}}, \quad X=-\left[u_{n}+\frac{1}{2 \hat{\beta}} u_{n}^{2}\right] \partial_{u_{n}}+t \partial_{t} .
$$

6. $K=\left(\begin{array}{ccc}1 & 0 & 0 \\ \hat{\beta} & \hat{\beta}^{2} & 0 \\ \hat{\beta}^{2} & \hat{\beta}^{3} & \hat{\beta}^{4}\end{array}\right), \quad \hat{\beta} \neq 0$,

$$
\begin{aligned}
& \dot{u}_{n}=\frac{u_{n}^{2} u_{n+1} u_{n-1}+\hat{\beta}\left(u_{n}^{2}+\hat{\beta} u_{n}\right)\left(u_{n+1}+u_{n-1}\right)+\hat{\beta}^{2}\left(u_{n}^{2}+2 \hat{\beta} u_{n}+\hat{\beta}^{2}\right)}{u_{n+1}-u_{n-1}}, \\
& X=\left[\hat{\beta}^{2}+\hat{\beta} u_{n}+u_{n}^{2}\right] \partial_{u_{n}} .
\end{aligned}
$$

Branch 2. $\alpha=0, \beta=1, \gamma=0, \beta+\hat{\beta}=0, \delta+\hat{\delta}=0, \hat{\gamma}+2 \lambda=0, \omega=0$.

$$
\begin{aligned}
\text { 7. } & =\left(\begin{array}{ccc}
0 & 1 & 0 \\
-1 & \lambda & \delta \\
-2 \lambda & -\delta & 0
\end{array}\right), \quad(\lambda, \delta) \neq(0,0) \\
\dot{u}_{n} & =\frac{u_{n} u_{n+1} u_{n-1}+\left(-u_{n}^{2}+\lambda u_{n}+\delta\right)\left(u_{n+1}+u_{n-1}\right)-2 \lambda u_{n}^{2}-2 \delta u_{n}}{u_{n+1}-u_{n-1}}, \\
X & =\left[\delta+\lambda u_{n}+u_{n}^{2}\right] \partial_{u_{n}} .
\end{aligned}
$$

Branch 3. $\alpha=0, \beta=0, \gamma=1, \hat{\beta}=\delta=\hat{\delta}=\omega=0, \hat{\gamma}=1, \lambda=-1$.

We have $r_{1}=1, r_{2}=3$, so $\operatorname{dim} L=3$.

\section{II. $r_{1}=4, r_{2}=2$}

We again follow the 3 branches.

Branch 1. $\alpha=1, \beta=0$.

I1. $\gamma \neq 0$. We have

$$
\hat{M}_{2} \sim\left(\begin{array}{ccc}
1 & 0 & \lambda-\gamma \\
0 & 1 & -\frac{\delta}{\gamma}
\end{array}\right)
$$

and all other entries in the row reduced matrix $\hat{M}_{2}$ must vanish (because $r_{2}=2$ ). We obtain

$$
\begin{aligned}
& \hat{\gamma}-\gamma+\hat{\beta} \frac{\delta}{\gamma}=0, \quad-\hat{\delta}+\hat{\beta}(\lambda-\gamma)+\hat{\gamma} \frac{\delta}{\gamma}=0, \\
& \omega-(\lambda-\gamma)^{2}-\frac{\delta^{2}}{\gamma}=0, \quad \omega-(\lambda-\gamma)(\lambda-\hat{\gamma})-\hat{\delta} \frac{\delta}{\gamma}=0, \\
& (\gamma-\lambda)(\delta-\hat{\delta})=0, \quad \hat{\beta}=0, \quad \delta-\hat{\delta}+\hat{\beta}(\lambda-\gamma)=0 .
\end{aligned}
$$


Conditions (4.9) imply

$$
\hat{\beta}=0, \quad \delta=\hat{\delta} \neq 0, \quad \gamma=\hat{\gamma}, \quad \omega=(\lambda-\gamma)^{2}+\frac{\delta^{2}}{\gamma} .
$$

The result is:

$$
\begin{aligned}
& \text { 8. } K=\left(\begin{array}{ccc}
1 & 0 & \gamma \\
0 & \lambda & \delta \\
\gamma & \delta & (\lambda-\gamma)^{2}+\frac{\delta^{2}}{\gamma^{2}}
\end{array}\right), \\
&\left(2 \lambda \gamma^{2}+\delta^{2}, \delta[2 \gamma-\lambda], \delta\left[2 \gamma^{3}+\lambda^{2} \gamma-\lambda \gamma^{2}+\delta^{2}\right]\right) \neq(0,0,0), \\
& \dot{u}_{n}=\frac{\left(u_{n}^{2}+\gamma\right) u_{n+1} u_{n-1}+\left(\lambda u_{n}+\delta\right)\left(u_{n+1}+u_{n-1}\right)+\gamma u_{n}^{2}+2 \delta u_{n}+(\lambda-\gamma)^{2}+\frac{\delta^{2}}{\gamma^{2}}}{u_{n+1}-u_{n-1}}, \\
& X=(-1)^{n}\left[\gamma-\lambda+\frac{\delta}{\gamma} u_{n}+u_{n}^{2}\right] \partial_{u_{n} .}
\end{aligned}
$$

I2. $\gamma=0$. To have $r_{2}=2$ we must put $\hat{\beta}=\delta=0$ and also $\hat{\gamma}=\hat{\delta}=0$. Then we obtain

$$
\begin{aligned}
\text { 9. } & K=\left(\begin{array}{ccc}
1 & 0 & 0 \\
0 & \lambda & 0 \\
0 & 0 & \omega
\end{array}\right), \quad \omega \neq \lambda^{2}, \\
\dot{u}_{n} & =\frac{u_{n}^{2} u_{n+1} u_{n-1}+\lambda u_{n}\left(u_{n+1}+u_{n-1}\right)+\omega}{u_{n+1}-u_{n-1}}, \quad X=(-1)^{n} u_{n} \partial_{u_{n}} .
\end{aligned}
$$

The branches II $(\beta=1, \alpha=\gamma=\beta+\hat{\beta}=\delta+\hat{\delta}=\hat{\gamma}+2 \lambda=\omega=0)$ and III $(\alpha=\beta=\hat{\beta}=$ $\delta=\hat{\delta}=\gamma+\lambda=\hat{\gamma}+\lambda=\omega=0, \gamma=1$ ) do not yield any new result.

\section{Conclusions}

What we mean by "integrable" was defined in the introduction. Thus an equation of the EYdKN family is integrable if and only if it satisfies (1.3), i.e. it is of the YdKN type.

It follows from the previous analysis that the symmetry algebra $L$ of the EYdKN equation satisfies $1 \leq \operatorname{dim} L \leq 5$. The largest dimension, namely 5 , is achieved for the equation (4.1). This is an integrable equation and in addition to point symmetries it allows higher symmetries. The two equations with four-dimensional Lie algebras, (4.2) and (4.6), are also both integrable. Of the three equations with three-dimensional symmetry algebras, (4.3) and (4.4) are integrable but (4.5) is not. Among the nine equations with two dimensional symmetry algebras only (4.8), (4.10) and (4.11) are integrable (they possess higher symmetries) for all values of the parameters involved.

We see that integrable equations, i.e. those in the YdKN class, rather than in the EYdKN one, tend to have larger Lie point symmetry algebras than the nonintegrable ones. This is however not a reliable integrability criterion. Indeed the nonintegrable equation (4.5) has a three-dimensional symmetry algebra whereas the generic integrable equation in the class $\mathrm{YdKN}$ class with $\alpha \neq 0$ has only the one symmetry $X_{0}=\partial_{t}$ (specifically an equation with $\alpha=1, \beta=0, \lambda \neq 2 \gamma$, $\left.\omega \neq \gamma\left(\lambda-\gamma^{2}+\delta^{2}\right)\right)$.

This rather loose relation between Lie point symmetries and integrability was already observed in a symmetry analysis of Toda type equations [12].

A complete symmetry analysis of the integrable GYdKN equation (1.4), (1.5) is not attempted here. We will just present one non-trivial example. Work is in progress to provide a complete classification. 
An example of the GYdKN equation is

$$
\dot{u}_{n}=\frac{\chi_{n+1}\left(u_{n+1}+u_{n-1}\right)+2 \chi_{n} u_{n}}{u_{n+1}-u_{n-1}}, \quad \chi_{n}=\frac{1+(-1)^{n}}{2}, \quad \chi_{n+1}=\frac{1-(-1)^{n}}{2} .
$$

Using the same approach as in Sections 3 and 4 above, we find that the symmetry algebra is four-dimensional with basis

$$
X_{0}=\partial_{t}, \quad X_{1}=t \partial_{t}+u_{n} \partial_{u_{n}}, \quad X_{2}=\chi_{n+1} \partial_{u_{n}}, \quad X_{3}=\chi_{n} u_{n} \partial_{u_{n}} .
$$

This is a direct sum $\left\{X_{0}, X_{1}, X_{2}\right\}+\left\{X_{3}\right\}$, where $\left\{X_{0}, X_{1}, X_{2}\right\}$ is solvable with $\left\{X_{0}, X_{2}\right\}$ as its Abelian nilradical.

The limit from the discrete equations considered in this article to the usual (continuous) Krichever-Novikov equation is quite complicated (see Section 2) and does not preserve symmetries. From Table 1 we see that the largest symmetry algebra is obtained for $f(u)=0$ and satisfies $\operatorname{dim} L=6$. Equation (2.1) in this case remains nontrivial. It is just the Schwarzian KdV equation $[6,19,25]$. A discrete analogue in this case would be $P=Q=R=0$ in (1.6), (1.7). This equation is trivial, the symmetry algebra is infinite-dimensional generated by

$$
X(\tau)=\tau(t) \partial_{t}, \quad U\left(\phi_{n}\right)=\phi_{n}\left(u_{n}\right) \partial_{u_{n}}
$$

where $\tau(t)$ and $\phi_{n}\left(u_{n}\right)$ are arbitrary $\left(C^{\infty}\right)$ functions of their arguments.

The Lie algebra element $P_{1}=\partial_{x}$, generating space translations, is always absent in the discrete case. Formally we can add the operator $\hat{N}=\partial_{n}$ to the symmetry algebra, as was done previously for the Toda lattice $[11,12]$. This corresponds (formally) to introducing a (discrete) group transformation $n^{*}=n+N$ with the understanding that $N$ is an integer (a shift on the lattice). This symmetry allows us to consider a periodic EYdKN equation, or equivalently to restrict to a finite lattice.

Finally, let us just give some examples showing how the Lie point symmetries can be used to reduce the considered differential-difference equation to simpler equations. Consider (4.1) and its dilation subalgebra $X_{1}$. A solution invariant under the subgroup generated by $X_{1}$ will have the form $u_{n}=c_{n} t^{-1 / 2}$. Putting this into (4.1) we find that the coefficient $c_{n}$ must satisfy the nonlinear difference equation

$$
c_{n}=\frac{1}{2}\left[\frac{1}{c_{n+1}}-\frac{1}{c_{n-1}}\right], \quad u_{n}=\frac{c_{n}}{\sqrt{t}} .
$$

Similarly the subalgebra $X_{3}+a X_{0}$ leads to the invariant solution

$$
u_{n}=\frac{a}{c_{n}-t}, \quad c_{n}=-\frac{a^{2}}{2} n+\beta .
$$

Work is in progress for a complete study of the group invariant solutions for all the invariant equations obtained in this article.

\section{Acknowledgements}

The research of L.D. has been partly supported by the Italian Ministry of Education and Research, PRIN "Continuous and discrete nonlinear integrable evolutions: from water waves to symplectic maps". The research of P.W. was partly supported by a research grant from NSERC of Canada. R.I.Y. has been partially supported by the Russian Foundation for Basic Research (grant numbers 10-01-00088-a and 11-01-97005-r-povolzhie-a). 


\section{References}

[1] Adler V.E., Bäcklund transformation for the Krichever-Novikov equation, Int. Math. Res. Not. 1998 (1998), no. 1, 1-4, solv-int/9707015.

[2] Adler V.E., Shabat A.B., Yamilov R.I., Symmetry approach to the integrability problem, Theoret. and Math. Phys. 125 (2000), 1603-1661.

[3] Bruzon M.S., Gandarias M.L., Classical and nonclassical reductions for the Krichever-Novikov equation, in ICNAAM 2010: International Conference of Numerical Analysis and Applied Mathematics, AIP Conf. Proc. 1281 (2010), 2147-2150.

[4] Dorodnitsyn V., Applications of Lie groups to difference equations, Differential and Integral Equations and Their Applications, Vol. 8, CRC Press, Boca Raton, FL, 2011.

[5] Dubrovin B., Krichever I.M., Novikov S.P., Integrable systems. I, Current Problems in Mathematics. Fundamental Directions, Vol. 4, VINITI, Moscow, 1985, 179-284 (in Russian).

[6] Güngör F., Lahno V.I., Zhdanov R.Z., Symmetry classification of KdV-type nonlinear evolution equations, J. Math. Phys. 45 (2004), 2280-2313, nlin.SI/0201063.

[7] Krichever I.M., Novikov S.P., Holomorphic bundles over algebraic curves and non-linear equations, Russ. Math. Surv. 35 (1980), no. 6, 53-80.

[8] Krichever I.M., Novikov S.P., Holomorphic fiberings and nonlinear equations. Finite zone solutions of rank 2, Sov. Math. Dokl. 20 (1979) 650-654.

[9] Levi D., Petrera M., Continuous symmetries of the lattice potential KdV equation, J. Phys. A: Math. Theor. 40 (2007), 4141-4159, math-ph/0701079.

[10] Levi D., Petrera M., Scimiterna C., Yamilov R.I., On Miura transformations and Volterra-type equations associated with the Adler-Bobenko-Suris equations, SIGMA 4 (2008), 077, 14 pages, arXiv:0802.1850.

[11] Levi D., Winternitz P., Continuous symmetries of discrete equations, Phys. Lett. A 152 (1991), 335-338.

[12] Levi D., Winternitz P., Symmetries of discrete dynamical systems, J. Math. Phys. 37 (1996), 5551-5576.

[13] Levi D., Winternitz P., Continuous symmetries of difference equations, J. Phys. A: Math. Gen. 39 (2006), R1-R63, nlin.SI/0502004.

[14] Levi D., Winternitz P., Yamilov R.I., Lie point symmetries of differential-difference equations, J. Phys. A: Math. Theor. 43 (2010), 292002, 14 pages, arXiv:1004.5311.

[15] Levi D., Yamilov R.I., Conditions for the existence of higher symmetries of evolutionary equations on the lattice, J. Math. Phys. 38 (1997), 6648-6674.

[16] Levi D., Yamilov R.I., Generalized symmetry integrability test for discrete equations on the square lattice, J. Phys. A: Math. Theor. 44 (2011), 145207, 22 pages, arXiv:1011.0070.

[17] Mokhov O.I., Canonical Hamiltonian representation of the Krichever-Novikov equation, Math. Notes 50 (1991), 939-945.

[18] Nijhoff F.W., Capel H., The discrete Korteweg-de Vries equation, Acta Appl. Math. 39 (1995), $133-158$.

[19] Nijhoff F.W., Hone A., Joshi N., On a Schwarzian PDE associated with the KdV hierarchy, Phys. Lett. A 267 (2000), 147-156, solv-int/9909026.

[20] Novikov D.P., Algebraic-geometric solutions of the Krichever-Novikov equation, Theoret. and Math. Phys 121 (1999), 1567-1573.

[21] Novikov S.P., Manakov S.V., Pitaevsky L.P., Zakharov V.E., Theory of solitons. The inverse scattering method, Contemporary Soviet Mathematics, Plenum, New York, 1984.

[22] Rasin O.G., Hydon P.E., Symmetries of integrable difference equations on the quad-graph, Stud. Appl. Math. 119 (2007), 253-269.

[23] Sokolov V.V., On the Hamiltonian property of the Krichever-Novikov equation, Sov. Math. Dokl. 30 (1984), 44-46.

[24] Svinolupov S.I., Sokolov V.V., Yamilov R.I., On Bäcklund transformations for integrable evolution equations, Soviet Math. Dokl. 28 (1983), 165-168.

[25] Weiss J., The Painlevé property for partial differential equations. II. Bäcklund transformation, Lax pairs, and the Schwarzian derivative, J. Math. Phys. 24 (1983), 1405-1413.

[26] Winternitz P., Symmetries of discrete systems, in Discrete Integrable System, Editors B. Grammaticos, Y. Kosmann-Schwarzbach and T. Tamizhmani, Lecture Notes in Phys., Vol. 644, Springer, Berlin, 2004, 185-243, nlin.SI/0309058. 
[27] Xenitidis P.D., Integrability and symmetries of difference equations: the Adler-Bobenko-Suris case, in Proc. 4th Workshop "Group Analysis of Differential Equations and Integrable Systems", University of Cyprus, Nicosia, 2009, 226-242, arXiv:0902.3954.

[28] Xenitidis P.D., Symmetry algebra of discrete KdV equations and corresponding differential-difference equations of Volterra type, arXiv:1105.4779.

[29] Xenitidis P.D., Papageorgiou V.G., Symmetries and integrability of discrete equations defined on a blackwhite lattice, J. Phys. A: Math. Theor. 42 (2009), 454025, 13 pages, arXiv:0903.3152.

[30] Yamilov R.I., Classification of discrete evolution equations, Uspekhi Mat. Nauk 38 (1983), no. 6, 155-156 (in Russian).

[31] Yamilov R.I., Symmetries as integrability criteria for differential difference equations, J. Phys. A: Math. Gen. 39 (2006), R541-R623. 